-Editorial·

\title{
Alzheimer's disease: from molecule to clinic
}

\author{
Jian-Zhi Wang* \\ Department of Pathophysiology, Key Laboratory of Neurological Diseases of The Ministry of Education, Tongji Medical \\ College, Huazhong University of Science and Technology, Wuhan 430030, China \\ *Guest Editor of the Special Issue, Associate Editor. E-mail: wangjz@mails.tjmu.edu.cn
}

(C) Shanghai Institutes for Biological Sciences, CAS and Springer-Verlag Berlin Heidelberg 2014

Alzheimer's disease (AD) is the most common neurodegenerative disorder and is characterized pathologically by the progressive intracellular accumulation of neurofibrillary tangles and extracellular deposition of plaques in the brain. Since hyperphosphorylated tau and $\beta$-amyloid $(A \beta)$ are respectively the major components of the tangles and the plaques, recent studies have mainly focused on the alterations associated with these two molecules. Clinically, AD features memory deterioration. More than 6 million patients in China alone are suffering from this devastating disease, and this figure is increasing with the acceleration of population ageing. Although many specific aspects of AD have been documented, the disease is hard to diagnose in the early stage and the efficacy of current treatments is very limited. Therefore, AD is attracting worldwide attention.

To help scientists and clinicians in the field better understand $A D$, we have organized this special issue. The 18 papers include 3 original research articles and 15 reviews invited from 17 laboratories in China, the United States, Canada, and Sweden. The content of this special issue covers the most recent progress in understanding the epidemiology, molecular pathogenesis, and potential diagnostic and therapeutic strategies of $A D$.

$A D$ is a multi-factorial disorder and the etiological factors are not fully understood. Over $95 \%$ of cases are sporadic in nature with onset in people over 65 years old, suggesting that aging is an independent risk factor for AD. To explore how aging is involved in AD neuronal loss, He's group demonstrates that uric formaldehyde is negatively correlated with cognitive abilities in older adults, and the increased formaldehyde also occurs in aged cell cultures. Furthermore, treatment of cells with certain concentrations of formaldehyde reduces cell viability and damages cell morphology ${ }^{[1]}$. Several microRNAs are implicated in aging. Zhang's group shows that miR-206 is upregulated in the hippocampus, cerebrospinal fluid, and plasma of APP/PS1 transgenic mice, and this downregulates the expression of brain-derived neurotrophic factor ${ }^{[2]}$. By intracellular injection of $A \beta_{42}$ in Drosophila, Huang's group reveals that the intraneuronal accumulation of $A \beta_{42}$ induces an age-dependent slowdown of neuronal transmission with a reduced transmission velocity of neuronal signals in the giant fiber system ${ }^{[3]}$. These new findings suggest that improving brain metabolism by targeting specific microRNAs, formaldehyde, and $A \beta_{42}$ during aging may be tractable strategies to protect neurons from degeneration.

Since there is, as yet, no efficient way to repair degenerating neurons, early diagnosis is critical for preventing or delaying neurodegeneration in AD. To achieve early diagnosis, longitudinal population-based studies on the application of non-invasive techniques and biomarkers are indispensable. Pei and Wang have analyzed 25 published studies of elderly Chinese residents from Mainland China, Hong Kong, Taiwan, and Singapore. The results show that the prevalence, incidence, and risk factors for dementia in Chinese populations are comparable to those in other ethnic groups, but no conclusive results were found for the prognosis of dementia ${ }^{[4]}$. Application of multi-modal imaging has recently shown great potential for the non-invasive clinical screening and diagnosis of $A D$. Han introduces each of the magnetic resonance imaging modalities used in longitudinal studies of $A D$ and discusses the future direction for these types of study ${ }^{[5]}$. By focusing specifically on changes in the topological organization of large-scale structural and functional brain networks, He 
proposes that $A D$ is associated with disrupted segregation and integration in the networks, and brain connectomics studies may uncover imaging markers for clinical diagnosis and for evaluating treatment of $A D^{[6]}$. As for cerebrospinal fluid biomarkers, Yang summarizes the novel candidates identified by highly-sensitive and high-throughput proteomic techniques in addition to the previously recognized $A \beta$ and tau proteins. They also provide perspectives on how biomarker discovery for $A D$ is evolving ${ }^{[7]}$.

Deregulation of brain metabolism plays an important role in the initiation and progression of AD. For instance, reduction of insulin, insulin receptor binding, or downstream signaling responsiveness has been reported in the AD brain. Gong reviews the important role of brain insulin deficits in the development of sporadic $A D$, and promising therapeutic implications by targeting the brain insulin pathway for the treatment of $A D$ and other neurodegenerative disorders ${ }^{[8]}$. Abundant studies show that oxidative stress is an essential process in AD pathogenesis, promoting $A \beta$ deposition, tau hyperphosphorylation, and the subsequent loss of synapses and neurons. Zhu and Zhong analyze how oxidative stress or its synergistic effects along with other environmental and metabolic factors may affect neuronal functions and cause neuronal failure ${ }^{[9-11]}$. Cholinergic hypofunction is an early pathological feature of AD. Zhang reviews recent progress in understanding the functional involvement of the M1 muscarinic acetylcholine receptor in $A D$ and in developing specific agonists for $A D$ treatment ${ }^{[12]}$. Although deficient autophagy has been reported in $A D$, stimulation of autophagy is not always beneficial. By summarizing the recent publications on autophagy in AD, Jia emphasizes that, during the implementation of autophagy modulation therapies, the nature of the autophagic defect, the timing of intervention, and the optimal level and duration of modulation should be considered $^{[13]}$. Recent genome-wide association studies demonstrate that neuroinflammation and lipid deregulation play crucial roles in $A D$. Zhang reviews the literature on the roles, mechanisms, and interrelations of neuroinflammation with $A \beta$ and apolipoprotein $E$ alleles ${ }^{[14]}$. Liu summarizes the involvement of different lipids in $A D^{[15]}$.

The tau abnormality alone can cause neurodegeneration and cognitive impairments that have been detected in over twenty neurodegenerative disorders, thus it has attracted widespread interest as a target protein. Alonso discusses the biological functions of tau in microtubule assembly and stabilization and its interaction with other proteins. She also analyzes how different posttranslational modifications of tau change its biological functions and the possible approaches to target these alterations ${ }^{[16]}$. The adult human brain expresses six isoforms of tau by alternative splicing of its pre-mRNA at exons 2, 3, and 10. Liu demonstrates that abnormal regulation of tau exon 10 splicing causes neurodegeneration, and reviews the regulation of this splicing by cis-elements and transfactors $^{[17]}$. It is widely believed that tau hyperphosphorylation causes neurodegeneration, however it is not clear how. Based on the new finding that tau regulates cell viability, Wang proposes that the nature of $A D$ neurodegeneration is the consequence of aborted apoptosis induced by tau hyperphosphorylation, therefore, proper manipulation of tau phosphorylation could be promising in arresting $A D$ neurodegeneration ${ }^{[18]}$.

Taken together, the papers presented in this special issue cover the wide spectrum of $A D$. We hope that this will promote new studies that provide a better understanding of this disease, and more importantly, in developing novel molecular and cellular approaches to effectively prevent it or at least delay its progression.

\section{REFERENCES}

[1] Yu J, Su T, Zhou T, He Y, Lu J, Li J, He R. Uric formaldehyde levels are negatively correlated with cognitive abilities in healthy older adults. Neurosci Bull 2014, 30(2): 172-184.

[2] Tian N, Cao Z, Zhang Y. MiR-206 decreases brain-derived neurotrophic factor levels in a transgenic mouse model of Alzheimer's disease. Neurosci Bull 2014, 30(2): 191-197.

[3] Lin JY, Wang WA, Zhang X, Liu HY, Zhao XL, Huang FD. Intraneuronal accumulation of $A \beta_{42}$ induces age-dependent slowing of neural transmission in Drosophila. Neurosci Bull 2014, 30(2): 185-190.

[4] Pei JJ, Giron MST, Jia J, Wang HX. Dementia studies in Chinese populations. Neurosci Bull 2014, 30(2): 207-216.

[5] Hu Z, Wu L, Jia J, Han Y. Advances in longitudinal studies of amnestic mild cognitive impairment and Alzheimer's disease based on multi-modal MRI techniques. Neurosci Bull 2014, 30(2): 198-206.

[6] Dai Z, He Y. Disrupted structural and functional brain connectomes in mild cognitive impairment and Alzheimer's disease. Neurosci Bull 2014, 30(2): 217-232.

[7] Sui X, Liu J, Yang X. Cerebrospinal fluid biomarkers of Alzheimer's disease. Neurosci Bull 2014, 30(2): 233-242. 
[8] Chen Y, Deng Y, Zhang B, Gong CX. Deregulation of brain insulin signaling in Alzheimer's disease. Neurosci Bull 2014, 30(2): 282-294.

[9] Bonda DJ, Wang X, Lee HG, Smith MA, Perry G, Zhu X. Neuronal failure in Alzheimer's disease: a view through the oxidative stress looking-glass. Neurosci Bull 2014, 30(2): 243-252.

[10] Chen Z, Zhong C. Oxidative stress in Alzheimer's disease. Neurosci Bull 2014, 30(2): 271-281.

[11] Wainaina MN, Chen Z, Zhong C. Environmental factors in the development and progression of late-onset Alzheimer's disease. Neurosci Bull 2014, 30(2): 253-270.

[12] Jiang S, Li Y, Zhang C, Zhao Y, Bu G, Xu H, Zhang YW. M1 muscarinic acetylcholine receptor in Alzheimer's disease. Neurosci Bull 2014, 30(2): 295-307.

[13] Liang JH, Jia JP. Dysfunctional autophagy in Alzheimer's disease: pathogenic roles and therapeutic implications. Neurosci Bull 2014, 30(2): 308-316.

[14] Dorey E, Chang N, Liu QY, Yang Z, Zhang W. Apolipoprotein $\mathrm{E}$, amyloid-beta, and neuroinflammation in Alzheimer's disease. Neurosci Bull 2014, 30(2): 317-330.

[15] Liu Q, Zhang J. Lipid metabolism in Alzheimer's disease. Neurosci Bull 2014, 30(2): 331-345.

[16] Beharry C, Cohen LS, Di J, Ibrahim K, Briffa-Mirabella S, Alonso Adel C. Tau-induced neurodegeneration: mechanisms and targets. Neurosci Bull 2014, 30(2): 346-358.

[17] Qian W, Liu F. Regulation of alternative splicing of tau exon 10. Neurosci Bull 2014, 30(2): 367-377.

[18] Wang JZ, Wang ZH, Tian Q. Tau hyperphosphorylation induces apoptotic escape and triggers neurodegeneration in Alzheimer's disease. Neurosci Bull 2014, 30(2): 359-366. 